\title{
Lidil
}

Revue de linguistique et de didactique des langues

47 | 2013

Le verbe pour exprimer le temps

Danièle Moore et Cécile Sabatier, Une semaine en classe en immersion française au Canada : le projet CECA au Canada

Grenoble, PUG, 2012, 144 p.

Marielle Rispail

(2) OpenEdition

Journals

Édition électronique

URL : http://journals.openedition.org/lidil/3280

DOI : $10.4000 /$ lidil.3280

ISSN : $1960-6052$

Éditeur

UGA Éditions/Université Grenoble Alpes

Édition imprimée

Date de publication : 31 mai 2013

Pagination : $172-174$

ISBN : 978-2-84310-247-9

ISSN : $1146-6480$

Référence électronique

Marielle Rispail, « Danièle Moore et Cécile Sabatier, Une semaine en classe en immersion française au

Canada : le projet CECA au Canada », Lidil [En ligne], 47 | 2013, mis en ligne le 01 décembre 2014,

consulté le 22 septembre 2020. URL : http://journals.openedition.org/lidil/3280 ; DOI : https://doi.org/ 10.4000/lidil.3280

(c) Lidil 


\section{Danièle Moore et Cécile Sabatier, Une semaine en classe en immersion française au Canada : le projet CECA au Canada, Grenoble, PUG, 2012, 144 p.}

Cet ouvrage est à la fois modeste et ambitieux : il se situe à Vancouver, en Canada anglophone, et veut rendre compte, jour après jour, heure après heure quasiment, d'un enseignement immersif en français. Projet apparemment limité, dans l'espace et le temps, à visée descriptive, mais dont on verra qu'il déborde largement de son cadre de départ. La recherche dont il rend compte a pris place dans le projet $\mathrm{CECA}^{1}$, entreprise conjointe de l'AUF, du CRAPEL et de la FIPF.

Francine Cicurel souligne dans sa préface que cette recherche, animée par Danièle Moore et Cécile Sabatier, met la focale sur l'échange lié aux problèmes de langue, ce qui amène les acteurs (enseignants et jeunes élèves) à insister sur le méta, dans leurs discours, quelles que soient les activités déployées. Cette dimension réflexive est sans doute à la fois un des axes transversaux et continus des pratiques observées, et ce qui fait le lien méthodologique et thématique entre les activités et interactions des enseignants et des apprenants pour les chercheuses.

Le projet n'est pas de construire une modélisation, de donner des leçons, mais de montrer ce qui se passe dans une classe ordinaire, comment on y travaille, pour donner des idées, susciter des envies, suggérer des transferts. Il n'y a pas de pratique exemplaire, il n'y a que des observations fines et sereines à faire, des expériences à partager, dans une attention sans relâche. À la portée de tout le monde, finalement? Ou presque... Car ces observations prennent forme peu à peu dans des analyses qui aident à comprendre ce qui se passe et comment, dans une posture de base et un protocole qui «refusent l'asymétrie enseignant/chercheur», car le chercheur vient apprendre.

Les auteures reviennent plusieurs fois dans l'ouvrage sur la méthodologie, à laquelle un long passage (p. 120 et suivantes) est pourtant consacré. Les choix effectués permettent (p. 27) de «mettre en tension l'articulation entre la forme plurielle de l'observation et les enjeux de cette observation». Une «combinaison de méthodes et d'outils d'observation d'une situation in situ, de l'enseignant et des élèves» permet de rassembler enregistrements vidéos, clichés photos, notes de terrain, entretiens d'explicitation, recueil de travaux d'élèves, etc. On escompte

1. Cultures d'enseignement, cultures d'apprentissage. 
donc passer, grâce à la description, d'une compréhension de la pratique enseignante par l'observation de praticiens expérimentés à une interrogation sur les contenus de formation. L'approche se veut empirique, contextuelle, naturaliste, participante.

Tout d'abord la classe est analysée selon trois axes : elle est à la fois une organisation sociale (p. 13 et suivantes), un espace spatial et temporel qui devient ensuite un espace de rencontres. Et aucun de ces axes n'est indépendant des autres. Dans le contexte ainsi dessiné, peuvent se forger des outils de formation et de recherche, définis par deux niveaux de lecture et d'écoute, complémentaires et dissociés : ce que font les enseignantes / ce que font les chercheuses. Dans les deux cas, on travaille «à hauteur d'enfants», les photos nous le prouvent, et ce déplacement du regard adulte permet de construire/déconstruire l'espace-classe, les savoirs, les relations entre acteurs, les stratégies d'apprentissage. Il met en valeur ces petits riens de la classe qui tissent la journée : les routines, les rituels, les menus du jour, car les chercheuses désirent «se pencher sur les évènements qui construisent le quotidien» (p. 75) et construire de la fierté. Chaque fois elles se demandent, sans à priori : comment ça se passe ? comment ça fonctionne ? qu'est-ce qui nous est donné à voir? à entendre ? Cette démarche «écologique», non interventionniste, nous avait déjà été montrée par Jean-Louis Dufays, dans l'ouvrage Gros plan sur la classe de français ${ }^{2}$, où il décrivait le quotidien de deux enseignantes de français en Belgique, après une année d'observation dans leurs classes. À la différence près qu'à Vancouver (cf. le contexte décrit dans le chapitre 2, riche et fouillé), l'environnement parle anglais (ou américain?) et qu'il s'agit de faire de la classe un milieu à la fois ouvert et francophone : d'où l'affiche «ici on parle français » à l'entrée et une hiérarchisation des priorités qui fait arriver le savoir seulement en $3^{\mathrm{e}}$ position dans le «vivre ensemble» de la classe. Autant dire qu'il n'est pas question d'«objectivité» dans cette recherche, mais plutôt de la construction patiente d'une compétence théorique qu'on pourrait appeler «mise en alerte». À force de regarder et de montrer comment fonctionne «l'agir enseignant», comment s'affinent les «gestes du métier», on fait œuvre de formation :

2. Cf. «De la discipline déclarée à la discipline apprise : un an d'observation de deux enseignantes de français et de leurs élèves en classe de $5^{\mathrm{e}}$ secondaire», dans M. Rispail et C. Ronveaux (dir.), Gros plan sur la classe de français, Peter Lang, 2010. 
formation non fondée sur la répétition d'un modèle mais sur une réflexivité aiguisée chaque jour, dans la voie ouverte par Cicurel, CambraGiné, Altet. L'outillage théorique est d'autant plus fort qu'il est peu étalé (Bucheton, Jorro, Vigner, etc.).

Cette réflexivité s'applique aussi envers les apprenants, car tout se construit selon l'idéologie de la redistribution : comment lier savoirs linguistiques et savoirs conversationnels? à propos de quels moments sociaux? (cf. le rituel du calendrier par exemple), et se répercute sur le travail des stagiaires dans la classe, qui voient peu à peu se transformer leur regard, qui révisent leurs positions critiques à priori. On comprend enfin qu'enseigner est un métier qui s'apprend.

L'approche ethnographique mise en place combine les pratiques et interactions dans la classe, la construction d'une culture éducative locale et interroge en même temps l'acte d'enseigner et l'acte d'apprendre. Elle amène à quelques découvertes inattendues : le statut de l'oral en immersion précoce, jamais dissociée de sa forme écrite, la dictée comme pratique innovante, le champ de la numératie, la place de la musique et des chansons, etc.

On met au jour la complexité de l'acte didactique (p. 29) et sa dynamique qui articule savoirs et actions. Les dix principes finaux qui concluent l'ouvrage n'apprendront rien à certain-e-s, mais il est réconfortant d'y trouver la pédagogie différenciée, l'évaluation formative, l'élève au centre, la classe comme espace de vie et comme «microsystème social». L'éthique fondamentale qui se dégage de la recherche confirme l'idée que cette étude, autant dans sa méthode que dans ses conclusions, peut être d'un apport certain dans d'autres contextes : immigration, enseignement précoce, enseignement hors immersion, langues minoritaires. Elle met par ailleurs au second plan quelques inquiétudes suscitées par la lecture : pourquoi des femmes seulement dans les enseignantes et les chercheuses? et quelles sont les langues d'origine des élèves, que le contrat «on parle français» pourrait faire exister malgré tout de façon explicite? Comment prendre en compte le plurilinguisme des élèves et des enseignantes (décrit p. 43)? On se demande si le tout-français craint l'aventure de la diversité...

Marielle Rispail CEDICLEC, Université Jean Monnet - St-Étienne LIDILEM, Université de Grenoble 